\title{
Do movement patterns differ between laboratory and field suction feeding behaviors in a Mexican cichlid?
}

\author{
Brook O. Swanson ${ }^{\mathrm{a}, \mathrm{c}}$, Alice C. Gibb ${ }^{\mathrm{a}}$, Jane C. Marks ${ }^{\mathrm{a}}$ \& Dean A. Hendrickson ${ }^{\mathrm{b}}$ \\ ${ }^{a}$ Department of Biological Sciences, Northern Arizona University, PO Box 5640, Flagstaff, Arizona, 86011, \\ USA (e-mail: Brook.Swanson@ucr.edu) \\ ${ }^{\mathrm{b}}$ Section of Integrative Biology, University of Texas, Patterson 521, UT, C0930Austin, TX, 78712, USA \\ ${ }^{\mathrm{c}}$ Department of Biology, University of California, Riverside, California, 92521, USA
}

Received 29 October $2004 \quad$ Accepted 30 May 2005

Key words: kinematics, functional morphology, Herichthys minckleyi

\section{Synopsis}

We analyzed feeding behavior of individuals of Herichthys minckleyi, the Cuatro Ciénegas cichlid, under laboratory conditions and freely behaving in their natural environment using high-speed video imaging. In a multivariate analysis of suction feeding behaviors there was no clear grouping of feeding events based on the environment, which suggests that most of the variability in the data was unrelated to differences between lab and field behaviors. In fact, the variability within an environment was far greater than the variability between the two environments. These results suggest that laboratory studies can accurately describe the kinematics of behaviors seen in the field. However, although lab based studies can quantify behaviors seen in the field, natural habitats are complex and provide individuals with the opportunity to exploit a wide range of food types and microhabitats, which may elicit behaviors not observed in the laboratory. However, feeding behaviors observed in the lab are representative of frequently used feeding behaviors in the field, at least for this species. Thus, we suggest that laboratory studies of feeding behavior, particularly those that test biomechanical or performance-based hypotheses can be extrapolated to natural environments.

\section{Introduction}

Feeding behavior is a critical aspect of how organisms interact with their environment because the ability to obtain food directly affects an organism's ability to survive and reproduce (Liem \& Osse 1975). Because of its pivotal role in individual survival, the mechanics of feeding have been examined in many animal species, and have received particular attention in teleost fishes (Lauder \& Reilly 1996). These studies have typically followed one of two patterns, either careful observation of behavior in the field (e.g. Tinbergen 1942), or controlled laboratory studies of the biomechanical basis of behavior (e.g. Lauder \&
Reilly 1996, Grubuch \& Wainwright 1997, Wainwright et al. 2001). These laboratory studies have elucidated the neurological basis for and performance consequences of different behavior modes, and have also been used to study the evolution of behavior.

One observation derived from these lab studies is that some teleost fishes can modulate their feeding behavior in response to different microhabitats and food types (Liem 1979, Liem 1980). Recent work demonstrates that this "modulatory multiplicity' is present in several families of teleosts; in each case, individuals adjust their feeding behavior in response to a change in food type or feeding context. For instance, the Labridae 
(Ferry-Graham et al. 2001) and the Tetraodontiformes (Turingan and Wainwright 1993) use different feeding modes when presented with food items that provide different challenges (e.g. fish use a more rapid suction feeding behavior when presented with elusive prey, and use a biting behavior when presented with attached prey). The Centrarchidae (Grubuch \& Wainwright 1997, Huskey 2004) modulate feeding behavior depending on the orientation of food or the habitat complexity that food is presented in (e.g. fish use a ram-based feeding behavior in open water and a suctionbased feeding behavior in more complex habitats). Not surprisingly, fishes not only change behavior type depending on context, but can also adjust a given feeding behavior. For instance, the kinematics of suction feeding can be adjusted depending on food type, orientation, and context (Ferry-Graham et al. 2001, Huskey 2004). This plasticity in behavior apparently allows fishes to feed successfully on a broad range of food items and within a wide range of microhabitats in the wild. However, it is not known whether behaviors observed in the laboratory are functionally equivalent to behaviors observed in the field.

Researchers have relied on lab studies to characterize feeding kinematics in teleost fishes because environmental variables including temperature, food type, predator orientation, and illumination can be controlled and movements (kinematics) of the feeding apparatus can be quantified (Alexander 1967, Liem 1979, Liem \& Kaufman 1984, Meyer 1989). However, few studies have measured feeding kinematics in freely behaving individuals in their natural habitats (Schmitt \& Coyer 1982, Ehlinger 1990). Because different laboratory conditions have been shown to produce different movement patterns, differences between laboratory and field environmental conditions may also be expected to result in distinct behaviors (Barlow 1968, Liem \& Summers 2000, Huskey 2004). These differences may lead to incorrect ecological or evolutionary conclusions if laboratory studies are extrapolated to draw conclusions about behavior in the wild.

In this study, we assess the similarity of feeding behaviors recorded in the laboratory and field to test the applicability of lab results to freely behaving individuals using the Cuatro Ciénegas cichlid, Herichthys minckleyi. This species is endemic to the Cuatro Ciénegas basin of north central Mexico, and demonstrates multiple prey-processing morphotypes (morphs) specialized to feed on different food resources (Kornfield \& Taylor 1983). In the pools and lakes they occupy, these fish have a broad diet, feed in multiple distinct microhabitats, and forage throughout the day (Smith 1982, Kornfield \& Taylor 1983, Liem \& Kaufman 1984). Here we use high-speed video and kinematic analyses to compare feeding behaviors in the field with behaviors in the laboratory. For suction feeding, one of the feeding behaviors, we compare movement patterns, or feeding kinematics across environments. We hypothesize that the differences in between the two environments will lead to different behaviors and distinct kinematic patterns. Alternatively, our null hypothesis is that behaviors and movements recorded in the lab are equivalent to those recorded in the field.

\section{Materials and methods}

\section{Field behavior}

Herichthys minckleyi inhabits small, clear, pools in the Cuatro Ciénegas basin of north, central Mexico. The small size and clarity of the pools allowed us to mark a large number of individuals within a habitat and locate them on subsequent days to record feeding behaviors. We conducted observations in two pools, Poza Mojarral Oeste and Poza Escobedo, and feeding events were recorded during June 2001.

We captured fish used for field studies with hook and line or gill nets and lightly anesthetized with clove oil (Munday \& Wilson 1997). Anesthetized fish were measured, weighed, and tagged with numbered pieces of plastic flagging $\left(1 \mathrm{~cm}^{2}\right)$ sutured to the epaxial musculature. The tags allowed the identification of individuals from the behavioral recordings and the absorbable dental sutures used to attach the tags softened and fell off after a week in the field, to minimize the impact of the study on the wild populations of $H$. minckleyi (Swanson et al. 2003). We tagged 30 fish in Poza Mojarral Oeste and 29 in Poza Escobedo (size range for all fish was 71-137 mm SL). After tagging and recovery from anesthesia, we released all individuals back into the pool. 
We allowed fish $24 \mathrm{~h}$ to recover from handling. After the recovery period, a researcher entered the pool and recorded all observable feeding events for marked individuals using a mixture of scan sampling and focal-animal sampling technique (Altmann 1974). During the observation period, we observed tagged fish foraging in a similar manner to un-tagged fish. In addition, individuals of this species rarely reacted to the presence of a researcher in the water, possibly because there are no known large predators in this system. Feeding events were recorded with a JVC GVL 9800 digital camcorder contained in a waterproof housing and recording at 240 fields-per-second. Foraging individuals were not recorded for longer than $1 \mathrm{~min}$ at a time to avoid bias due to interdependence of feeding observations. Identification numbers were generally not legible to the researcher in the field (although they could be read from the digital video during analysis). Thus, although feeding events were not sampled randomly, sampling of individuals was unbiased.

From the recordings of individual feeding events, we determined the individual identification number and feeding behavior type. Behavioral categories observed in the field were based on consistent differences in movement patterns and published kinematic descriptions (Swanson et al. 2003). Recordings of a sub-set of the feeding events were also used for quantitative analyses of feeding movements (e.g. kinematic analysis, see below). The feeding events chosen for this analysis represented recordings of the lateral view that were clear and where details of the morphology of the head were discernable.

\section{Laboratory behavior}

We used six individuals of $H$. minckleyi for laboratory feeding recordings. Fish used for this portion of the study were second-generation, captivebred and were maintained in individual, glass, 10-gallon aquaria with canister filters. The type of fish used (i.e. captive bred) for this portion of the study and the sample size were dictated by the difficulty in obtaining wild-caught individuals (Cuatro Ciénegas is a protected area and $H$. minckleyi is a protected species).

The room where fish were housed was maintained on a $14: 10$ light dark cycle at $25-30^{\circ} \mathrm{C}$. Fish remained in their home aquaria and were provided with several types of non-elusive prey items (cichlid chow pellets, fish flakes, blood worms, crushed and whole snails, and filamentous algae) during feeding trials. A JVC GVL 9800 digital camcorder was also used for laboratory video analysis to minimize differences between lab and field events based on frame rate, resolution or recording system. Multiple feeding events for each individual were recorded to digital tape until four feeding events of sufficient quality (body of fish normal to the camera, sufficient light to resolve jaw movements, etc.) were captured for each of four individuals. All feeding events were recorded from a lateral view, which allowed optimal observation of the movements of the upper and lower jaw, as well as movements of the cranium and the hyoid apparatus.

\section{Kinematic analysis}

Suction feeding behavior was the only behavior observed in the lab (see below); therefore, the kinematic analysis was constrained to a comparison of lab suction feeding behavior with field suction feeding events. Each frame from the digital video was de-interlaced using a program by Martyn Shorten, of BioMechanica, LLC. The frames were cut and recompiled into a series of consecutive images for analysis, resulting in 240 fields-persecond video.

The scale was set for digital measurements on the video images by using the standard length of each fish (taken using a measuring board out of the water). Landmarks (including jaw tips, rostral aspect of the orbit, ventral aspect of the hyoid, articulation between the lower jaw and the suspensorium, anterior-ventral end of the premaxilla, and dorsal aspect of the neurocranium) were chosen on the surface of the fish that defined morphological features important in feeding movements (Ferry-Graham et al. 2001). These landmarks were digitized in consecutive frames and position of the landmarks was used to calculate distance and timing variables during feeding events, including maximum gape $(\mathrm{mm})$, hyoid depression $(\mathrm{mm})$, premaxillary protrusion $(\mathrm{mm})$, cranial rotation $\left({ }^{\circ}\right)$, lower jaw angle $\left({ }^{\circ}\right)$, and time from first movement to maximum for each of these variables (s). 
A Principal Components Analysis (PCA), using the variables described above, was used to quantify sources of variance in the data and to visualize differences between lab and field suction feeding behavior in multivariate space. A multivariate analysis of variance (MANOVA) with the 10 measurement and timing variables from the PCA was used to test the null hypothesis that suction feeding is statistically indistinguishable between lab and field feeding events. Multiple one-factor analyses of variance were also used as post-hoc tests to compare each kinematic variable between lab and field feeding events.

\section{Results}

\section{Description of behaviors observed}

Feeding behavior in the field was variable and included a variety of distinct feeding patterns, as has been previously reported (Swanson et al. 2003). These behavior patterns included suction feeding (fish drew a food item into the mouth using a movement pattern consistent with suction feeding in other cichlids), scraping (fish pressed the upper and lower jaws against the substrate and abraded attached material off of the surface), diving (fish inserted a large portion of their bodies into the soft substrate at a high angle, retrieving material from below the surface of the substrate), and scooping behaviors (fish inserted a portion of the head into the soft substrate at a low angle and retrieved material off of the surface of the substrate; (Swanson et al. 2003). The suction feeding behavior occurred in all microhabitats, while the scraping behavior was only observed on hard substrates, and the scooping and diving behaviors were only observed in soft flocculent substrates. The suction feeding behavior was the most common behavior and accounted for $62 \%$ of total feeding events. However, under our lab conditions, fish only produced the suction feeding behavior. The observation of only a single behavior type in the laboratory constrained the kinematic comparison to field suction feeding vs. laboratory suction feeding.

\section{Kinematic comparisons}

In the principal components analysis of field and laboratory suction feeding events based on 10 measurement and timing variables, the first four principal components accounted for $82 \%$ of the variability in the suction feeding data set. The other principal components accounted for less than $6 \%$ of variance each and were not included in the analysis. There was no pattern of separation between the lab and field events for these first four principal components (Figure 1). This indicates that the majority of the variability in the data occurs within both the lab and field groups and is not due to differences between those groups. Additionally, we found no statistical difference in the kinematics of suction feeding between the laboratory and the field using the 10 kinematic variables (MANOVA $\mathrm{F}_{10,11}=1.46, \mathrm{p}=0.22$ ), and the means for all 10 kinematic variables were indistinguishable across environments (Figure 2). The differences introduced by the second generation, lab bred individuals should maximize differences in our comparisons. The conclusions of no difference between contexts are therefore conservative.

\section{Discussion}

Suction feeding is often regarded as the most primitive feeding behavior for aquatic vertebrates (Lauder \& Liem 1983, Lauder \& Reilly 1996). Almost all fishes, and many other aquatic vertebrates, employ suction feeding to capture prey (Aerts \& Vree 1993, Lemell et al. 2002, Carreño \& Nishikawa 2004). In addition, kinematic and electromyographic (i.e. muscle activity) data from a wide range of species suggest that the motor patterns generated by the central nervous system that are responsible for suction feeding in fishes are conserved across a range of taxa (Lauder \& Liem 1983). The conservation of these motor patterns, and the widespread use of this behavior across groups implies that this mechanism is important to the feeding ecology of many species.

In our study, suction feeding was the only feeding behavior observed for $H$. minckleyi in the laboratory and it was the most common behavior employed by the species in the field, occurring in a wide range of microhabitats (Swanson et al. 2003). Liem $(1975,1979$, 1980) suggested that suction feeding is the most efficient feeding behavior in cichlids, and can be employed to exploit a variety 

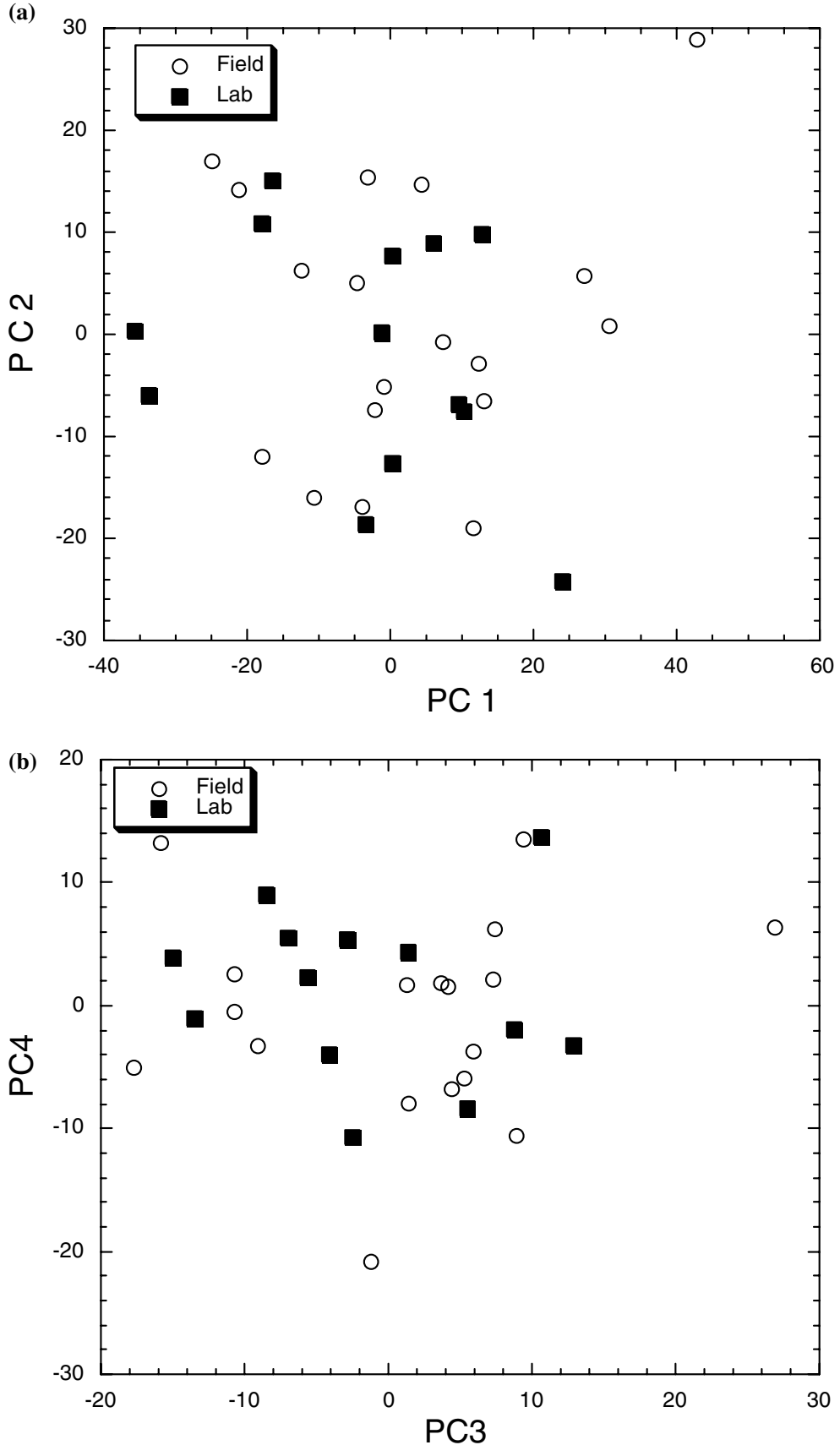

Figure 1. Plot of (a) the first two principal components and (b) principal components three and four for lab and field suction feeding events based on 10 measurement and timing variables. Each symbol represents an individual feeding event.

of food items. Herichthys minckleyi are known to include variety of food items in their diet (Smith 1982) and suction feeding may provide a general mechanism with which to capture a variety of prey.
In a multivariate description of the data, there was no clear grouping of feeding events based on the environment in which they occurred, which suggests that most of the variability in the data was unrelated to differences between lab and field 

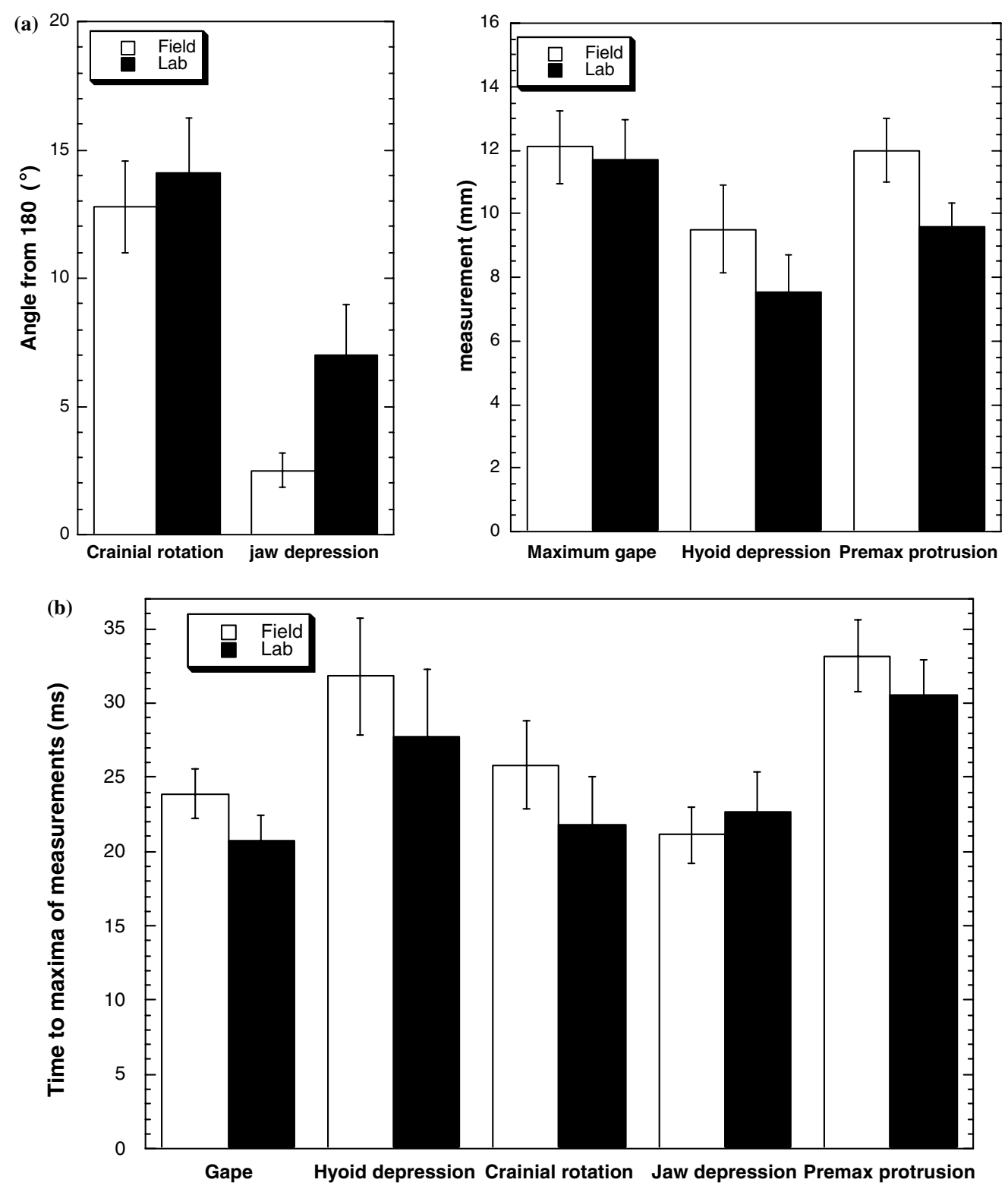

Figure 2. Means \pm 1 S.E.M for measurement (a) and timing variables (b) used in the principal components analysis and the multivariate analysis of variance for both lab and field suction feeding events in $H$. minckleyi.

behaviors. It is possible that there are differences in suction feeding behavior that we did not detect in this analysis; we chose these 10 movement variables because they are commonly used in kinematic analyses of feeding behavior. Because these variables describe head and jaw movements essential to producing suction (Ferry-Graham et al. 2001), we believe they should detect most of the variability related to suction performance. In fact, suction feeding events were variable. However, the variability within an environment exceeded the variability between the groups. This suggests that the kinematic variables we chose were sufficient to detect variation in feeding movements among feeding events, but that no significant variability was present between envi- 
ronments. It is also possible that the small sample size available for lab analysis did not have enough power to resolve differences. A power analysis (JMP software; not shown) indicated that with the observed differences in means we would have needed between 71 and 5000 individuals to resolve statistically significant differences. Therefore, we believe that any differences are small and are not functionally relevant. These similarities between behaviors in these very different contexts may also point to a constraint on the amount of plasticity possible in a complex behavior such as suction feeding. Sih et al. (2004) have suggested that complex suites of behavior can be evolutionarily constrained and more research needs to be conduced to identify how complex feeding behaviors fit into this framework.

To our knowledge, this study provides the first direct comparison of feeding jaw kinematics between lab and field contexts. The development of portable, high-speed, digital imaging systems has allowed us to take the lab to the animal rather than bringing the animal to the lab. This innovation allows quantitative studies of feeding kinematics to be conducted in an ecologically relevant context. Additionally, we can begin to test the validity of laboratory descriptions of feeding jaw kinematics, which have been historically important in the understanding of the evolution of feeding behavior. Our data indicate that studies of suction feeding for $H$. minckleyi are representative of the most common feeding behaviors performed in the field.

However, lab behavioral studies cannot mimic the natural range of food items and microhabitats observed in a species' natural habitat, even if animals are presented with a variety of food types. It is clear that a wider range of behaviors may be elicited in the lab by providing a wider range of food stimuli (Liem \& Summers 2000, Ferry-Graham et al. 2001, Huskey 2004). However, animals placed in captivity rapidly 'lose' many of their natural feeding modes. Liem \& Summers (2000) observed a decrease in the number of behavior modes demonstrated by cichlid species with increased time in captivity, even when complex and challenging foods were consistently presented. It appears that the predictability of non-elusive prey in captivity may enable fish to rely on a primitive, and highly efficient feeding behavior: suction feeding.
Barlow (1968) suggested that the modulation of behavioral motor patterns depends on a wide range of external stimuli and that behaviorists should use more precise, quantitative measurements to describe potentially undetected variations in behavior. We suggest that field high-speed imaging techniques allow studies of behavior that both occur with relevant external stimuli, and provide the resolution to discern the mechanisms behind complex behavior. We also suggest that functional morphologists should attempt to work in the field, or if this is not possible, with wild or recently captured animals placed in contexts that closely mimic natural habitats. However, we note that our data confirm that feeding behaviors observed in the lab are representative of frequently used behaviors in the field, at least for this species. Thus, although the breadth of behaviors observed in the lab probably does not represent the full range of behaviors used in the field, laboratory studies of biomechanical, neurological or performance-based hypotheses can be extrapolated to natural systems.

\section{Acknowledgements}

We thank K. Cummins, H. Kloppel, C. Williamson, M. Hess, and A. Cohen for help with fish capture in the field. We thank M. O'Neill, H. Hornstra, C. Pace, K. Salminin, for help with lab fish care. Three anonymous reviewers, D. L. G. Noakes and W. L. Montgomery provided comments on earlier versions of the manuscript. We give special thanks to M. Stephens for providing our laboratory stock of fish. M. Shorten of BioMechanica, LLC provided us with image processing software and A. Harrell and J. Meyers helped us with data analysis. This work was supported by a NAU intramural grant and NSF IBN 0002301 to ACG, EPA Star Fellowship U91591901 to BOS, an Ecosystem grant from The Nature Conservancy to JCM and DAH, and NSF DEB 0074876 POWRE grant to JCM.

\section{References}

Aerts, P. \& F.D. Vree. 1993. Feeding performance and muscular constraints in fish. J. Exp. Biol. 177: 129-147. 
Alexander, R.M. 1967. The functions and mechanisms of the protrusible upper jaws of some acanthopterygian fish. J. Zool. 151: 43-64.

Altmann, J. 1974. Observational study of behavior: sampling methods. Behavior 48: 227-265.

Barlow, G.W. 1968. Ethological units of behavior. pp. 217-232. In: D. Ingle, (eds.) The Central Nervous System and Fish Behavior, University of Chicago Press, Chicago.

Carreño, C.A. \& K.C. Nishikawa. 2004. Does Xenopus laevis use suction for prey capture? Integr. Comp. Biol. 44.

Ehlinger, T.J. 1990. Habitat choice and phenotype-limited feeding efficiency in Bluegill: Individual differences and trophic polymorphism. Ecology 71: 886-896.

Ferry-Graham, L.A., P.C. Wainwright, M.W. Westneat \& D.R. Bellwood. 2001. Modulation of Prey Capture Kinematics in the Cheeklined Wrasse Oxycheilinus digrammus (Teleostei: Labridae). J. Exp. Zool. 290: 88-100.

Grubuch, J.R. \& P.C. Wainwright. 1997. Motor basis of suction feeding performance in Largemouth Bass, Micropterus salmoides. J. Exp. Zool. 277: 1-13.

Huskey, S.H. 2004. Modulation of prey-capture kinematics in large-mouth bass, Micropterus spp. Integr. Comp. Biol. 44.

Kornfield, I. \& J.N. Taylor. 1983. A new species of polymorphic fish, Cichlasoma minckleyi, from Cuatro Ciénegas, Mexico (Teleostei: Cichlidae). Proceedings of the Biological Society of Washington. Washington DC 96: 253-269.

Lauder, G.V. \& K.F. Liem. 1983. The evolution and interrelationships of the actinopterygian fishes. Bull. Museum Comp. Zool. 150: 95-197.

Lauder, G.V. \& S.M. Reilly. 1996. The mechanistic bases of behavioral evolution: A multivariate analysis of musculoskeletal function. pp. 104-137. In: E.P. Martins, (eds.) Phylogenies and the Comparative Method in Animal Behavior, Oxford University Press, New York.

Lemell, P., C. Lemell, P. Snelderwaard, M. Gumpenberger, R. Wocheslander \& J. Weisgram. 2002. Feeding patterns of Chelus fimbriatus (Pleurodira: Chelidae). J. Exp. Biol. 205: 1495-1506.

Liem, K.F. 1979. Modulatory multiplicity in the feeding mechanism in cichlid fishes, as exemplified by the invertebrate pickers of Lake Tanganyika. J. Zool., London 189: 93-125.

Liem, K.F. 1980. Adaptive significance of intra- and interspecific differences in the feeding repertoires of cichlid fishes. Am. Zool. 20: 295-314.
Liem, K.F. \& L.S. Kaufman. 1984. Intraspecific macroevolution: Functional biology of the polymorphic cichlid species Cichlasoma minckleyi. pp. 203-215. In: I. Kornfield, (eds.) Evolution of Species Flocks, University of Maine at Orono Press, Orono, Maine.

Liem, K. \& J. Osse. 1975. Biological versatility, evolution, and food resource exploitation in African cichlid fishes. Am. Zool. 15: 427-454.

Liem, K.F. \& A.P. Summers. 2000. Integration of versatile functional design, population ecology, ontogeny and phylogeny. Netherlands J. Zool. 50: 245-259.

Meyer, A. 1989. Cost of morphological specialization: feeding performance of the two morphs in the trophically polymorphic cichlid fish, Cichlasoma citrinellum. Oecologia 80: 431436

Munday, P.L. \& S.K. Wilson. 1997. Comparative efficacy of clove oil and other chemicals in anaesthetization of Pomacentrus amboinensis, a coral reef fish. J. Fish Biol. 51: 931938

Schmitt, R.J. \& J.A. Coyer. 1982. The foraging ecology of sympatric marine fish in the genus Embiotoca (Emdiotocidae): Importance of foraging behavior in prey size selection. Oecologia 55: 369-378.

Sih, A., A.M. Bell, J.C. Johnson \& R.E. Ziemba. 2004. Behavioral syndromes: an integrative overview. Quart. Rev. Biol. 79: 241-274.

Smith, D.C. 1982. Trophic ecology of the cichlid morphs of Cuatro Ciénegas, Mexico. unpublished M.S. thesis, University of Maine at Orono, Orono.

Swanson, B.O., A.C. Gibb, J.C. Marks \& D.A. Hendrickson. 2003. Trophic polymorphism and behavioral differences decrease intra-specific competition in a cichlid, Herichthys minckleyi. Ecology 84: 1441-1446.

Tinbergen N. 1942. An objectivistic study of the innate behaviour of animals. Bibliotheca Biotheoretica: 39-98.

Turingan, R.G. and Wainwright, P.C. 1993. Morphological and functional bases of durophagy in the Queen triggerfish, Balistes vetula (Pisces, Tetraodontiformes). J. Morph. 215: 101-118.

Wainwright, P.C., L.A. Ferry-Graham, T.B. Waltzek, A.M. Carroll, C.D. Hulsey \& J.R. Grubich. 2001. Evaluating the use of ram and suction during prey capture by cichlid fishes. J. Exp. Biol. 204: 3039-3051. 